\title{
Paraoxonase-1 and Glutathione Peroxidase Activity as a Screening Tool in Detecting Severity of Coronary Artery Disease: Case Control Feasibility Study
}

\author{
Dinushka Wickramasinghe ${ }^{1}$, Hemantha Peiris ${ }^{1}$, Lal Gotabhaya Chandrasena ${ }^{2}$, Vajira Senaratne ${ }^{3}$, \\ Rasika Perera ${ }^{1, *}$ \\ ${ }^{1}$ Department of Biochemistry, Faculty of Medical Sciences, University of Sri Jayewardenepura, Gangodawila, Nugegoda, Sri Lanka \\ ${ }^{2}$ Nawaloka Hospitals, Colombo, Sri Lanka \\ ${ }^{3}$ Cardiology Unit, National Hospital Sri Lanka, Colombo 07, Sri Lanka
}

Email address:

dinushka15@gmail.com (D. Wickramasinghe), hempeiris@sjp.ac.lk (H. Peiris),dgmnh@sltnet.lk (L. G. Chandrasena), vajirasenaratne@gmail.com (V. Senaratne),rasika@sjp.ac.lk (R. Perera)

\section{To cite this article:}

Dinushka Wickramasinghe, Hemantha Peiris, Lal Gotabhaya Chandrasena, Vajira Senaratne, Rasika Perera. Paraoxonase-1 and Glutathione Peroxidase Activity as a Screening Tool in Detecting Severity of Coronary Artery Disease: Case Control Feasibility Study. American Journal of Biomedical and Life Sciences. Vol. 3, No. 6, 2015, pp. 120-126. doi: 10.11648/j.ajbls.20150306.14

\begin{abstract}
Glutathione peroxidase and paraoxonase-1 is reported to be a useful marker for monitoring cardiovascular disease. Due to the paucity of information on the association of above markers and severity of coronary artery disease in South Asian patients, case control study was performed with 85 patients (58 males and 27 females) 40-60 years of age confirmed as having coronary artery disease on coronary angiography findings and 85 age and sex matched healthy volunteers as controls. Blood samples were analyzed for serum paraoxonase-1 and erythrocyte Glutathione peroxidase activity in both groups and the severity of coronary artery disease was assessed using coronary angiographic scoring system based on vessel, stenosis and extent score. Patients with coronary artery disease showed significantly low paraoxonase-1 and Glutathione peroxidase activity compared to control subjects. However, according to the best cutoff value determined by receiver operating characteristic analysis for serum paraoxonase- $1(38 \mu \mathrm{g} / \mathrm{mL})$ did not show a significantly high sensitivity, negative predictive value and negative likelihood ratio when compared to erythrocyte Glutathione peroxidase $(84.5 \mathrm{U} / \mathrm{gHb})$ in predicting the severity of coronary artery disease assessed by three angiographic scores. Glutathione peroxidase appears to be an accurate marker in ruling out major coronary vessel disease and luminal narrowing by atheroma.
\end{abstract}

Keywords: Glutathione Peroxidase, Paraoxonase, Vessel Score, Stenosis Score, Extent Score

\section{Introduction}

Atherosclerosis is the major cause of coronary artery disease $(\mathrm{CAD})$ and some of the major risk factors for CAD are age gender, familial predisposition, smoking and hyperlipideamia. Although several hypotheses have been put forward to explain the mechanisms of atherosclerosis, most of these are directly or indirectly involve oxygen derived free radicals $[1,2]$. Oxygen derived free radicals are abundant in animal and human cells, they begin production via many enzymatic reactions. However, low levels of reactive oxygen species (ROS) serve as signaling molecules for metabolic regulation [3]. Oxidative stress may results when cellular antioxidant defense mechanisms are unable keep pace with detoxification of ROS. This results in lipid peroxidation of membrane lipids and lead to vascular endothelial injury and microvascular dysfunction [3]. Thus, balance between free radical generation and antioxidants activity is one of the major contributory factors for pathogenesis of atherosclerosis. Among antioxidant enzymes, glutathione peroxidase (GPx) constitutes a first line of defense against oxidative stress by removing key reactive oxygen species [4] whereas, PON-1, a calcium-dependent esterase, is largely responsible for the antioxidant and anti-inflammatory actions of High Density Lipoprotein (HDL) due to its ability to hydrolyze oxidized phospholipids and also being considered as an anti-atherogenic mediator [5]. Furthermore, both antioxidants have shown most convincing and statistically significant inverse associations with $\mathrm{CAD}[6,7]$. 
Recent meta-analysis studies assessing the association of GPx and PON-1 activity in cardiovascular disease show that there is substantial heterogeneity due to methodological and ethnic variability [4]. Hence, accurate assessments of these CAD risk markers are needed to substantiate their value in clinical medicine. To our knowledge these studies have concentrated mainly on the degree of coronary atherosclerosis as assessed by the number of major vessels involved in angiographic findings rather than quantifying the atherosclerosis process. Previous studies have used angiographic Gensini score in assessing the severity of coronary atheroma [8]. Thus, previous authors have not considered the proportion of the coronary artery tree involved by angiographycally detectable atheroma as assessed by the stenosis and extent scores [9, 10], which place emphasis on the number of vessels showing significant stenosis. Hence, this study considered all three above angiography scores which full fills this need in assessing extent of coronary atherosclerosis.

\section{Methods}

\subsection{Study Participants}

In this case control study the sample size was calculated for a matched case control study with a power of $80 \%$; ratio of cases to controls $1: 1$; exposure in controls $30 \%$; expected odds ratio of 2.6 and an alpha error of $5 \%$.

Thus, the present study was carried out with 85 patients (58 males and 27 females) aged 40-60 years who were confirmed as having CAD by coronary angiography findings at Cardiology Unit, National Hospital and Nawaloka Hospitals PLC, Colombo Sri Lanka during 2013 and 2014.

Subjects with a history of renal or hepatic disease, malignancy and those on statin therapy that could influence oxidative status were excluded from the study. A total of 85 age and sex matched healthy volunteers who had normal exercise ECG and estimated Glomerular Filtration Rate (eGFR) more than $60 \mathrm{ml} / \mathrm{min} / 1.73 \mathrm{~m}^{2}$ attending a routine health screening program at Family Health Care Centre, University of Sri Jayewardenepura, Nugegoda, Sri Lanka were recruited as controls to compare the PON-1 and GPx activities with patients.

\subsection{Collection of Samples and Biochemical Investigations}

Venous blood samples were drawn after an overnight fast (8-10 hours) from both patients and controls. Blood samples were immediately divided into two halves and one half was transferred into heparin coated tubes for GPx assay whilst the remaining was transferred into tubes without an anticoagulant for PON-1 assay.

Blood samples collected into lithium heparin tubes were immediately centrifuged at $3000 \mathrm{~g}$ for 5 minutes to separate the plasma and erythrocytes. Haemolysate prepared from prewashed erythrocytes with ice-cold isotonic $\mathrm{NaCl}$ were assayed for GPx. Serum samples were stored at $-80^{\circ} \mathrm{C}$ pending PON-1 analysis.

\subsection{Biochemical Assays}

\subsubsection{Glutathione Peroxidase}

Erythrocyte total GPx assay was carried out on the red blood cell hemolysate using Randox reagents (Randox Laboratories Limited, United Kingdom) on a Konelab 20XT Clinical Chemistry Auto Analyzer.

\subsubsection{Paraoxonase-1 (PON-1)}

PON-1 assay was carried out on serum using Paraoxonase1 ELISA assay kits (USCN life sciences Inc, Wuhan, China) with anti-human PON-1 monospecific antibodies and microplate reader (Bio-Rad 680) at $450 \mathrm{~nm}$.

\subsection{Assessment of Severity of Coronary Artery Disease}

Coronary angiography reports and the compact disc recordings of angiograms were independently reviewed by an interventional cardiologist, who had no access to the patients' clinical and laboratory findings. Angiogram findings were then evaluated using three different scoring systems - vessel score, stenosis score, and extent score. The angiograms were scored according to a method described by Sullivan et al 1990 [11] as given below. Identified coronary arteries were Main Left Coronary Artery, Left Anterior Descending Artery, Main Diagonal Branch, First Septal Perforator, Left Circumflex Artery, Obtuse Marginal and Posterolateral Vessels, Right Coronary Artery, Main Posterior Descending Branch. The scoring system is described below.

\subsubsection{Vessel Score}

"This was calculated as the number of vessels with a significant stenosis $(70 \%$ or greater reduction in lumen diameter). Depending on the number of vessels involved, vessel score ranged from 0 to 3 . The left main coronary artery stenosis was scored as single vessel disease".

\subsubsection{Stenosis Score}

"The stenosis score was calculated by a modified Gensini score as described by Reardon et al in 1985 [9] and Hamsten et al 1986 [10], which places emphasis on the severity of stenosis while including some measure of the extent of coronary artery disease. Briefly, the most severe stenosis in each eight coronary segments was graded according to severity, that is; a grade of 1 for $1-49 \%$ reduction in luminal diameter, 2 for $50-74 \%, 3$ for $75-99 \%$ and 4 for total occlusion. The scores for each of the eight segments were added together to give a total score out of a theoretical maximum of 32 ".

\subsubsection{Extent Score}

"The extent score was calculated according to the method described by Sullivan et al in 1990 [11] which indicates the proportional of the coronary artery tree involved by angiographycally detectable atheroma. The proportion of each vessel involved by atheroma, identified as luminal irregularity was multiplied by a factor for each vessel: left main artery, 5; left anterior descending artery, 20; main diagonal branch, 10; first septal perforate, 5; left circumflex 
artery, 20; obtuse marginal and postero-lateral vessels, 10; right coronary artery, 20; and main posterior descending branch, 10. When the major lateral wall branch was a large obtuse marginal on intermediate vessels, this was given a factor of 20 and the left circumflex artery a factor of 10 . When a vessel was occluded and the distal vessel not fully visualized by collateral flow, the proportion of vessel not visualized was given the mean extent score of the remaining vessels. The scores for each vessel or branch were added to give a total score out of 100 , being the percentage of the coronary intimal surface area involved by atheroma".

\subsection{Data Processing and Statistical Analysis}

Reference intervals for GPx and PON-1 in control subjects were determined using 95\% confidence intervals (Cl's). Owing to the skewed distribution of GPx and PON-1 activities, logarithmic transformations of these data were performed using SPSS software version 16.0 (Chicago, Illnois).

Multivariate logistic regression analysis was performed to eliminate the influence of confounding factors for CAD. Odds Ratio was calculated to assess risk of CAD in population. A p value $\leq 0.05$ was considered as statistically significant. Continuous variables were analyzed using independent sample $\mathrm{t}$ test, Analysis of variance (ANOVA) and Spearman correlations were calculated to determine the correlation between the coronary angiographic scores and GPx and PON1 levels. A 'p' value $\leq 0.05$ was considered to be significant.

Conventional coronary angiography has been considered as a gold standard for diagnosis of CAD. The diagnostic accuracy of PON-1 and GPx for CAD based on three scores was determined by measuring the area under the ROC curve (AUC), 95\% confidence interval, sensitivity (Sen\%), specificity (Spc\%), positive (PP\%) and negative (NP\%) predictive values, Positive (PLR) and negative (NLR) likelihood ratios. The accuracy of detecting the severity of CAD in patients using these two biomarkers was determined by measuring the area under the Receiver Operating Characteristics (ROC) curve, 95\% confidence interval, sensitivity, specificity and positive and negative predictive values and likelihood ratio using the conventional coronary angiography findings.

\subsection{Ethical Considerations}

Informed written consent was obtained from all participants before recruitment to the study. The study design and the study protocol was approved by the Ethics Review Committee of the Faculty of Medical Sciences, University of Sri Jayewardenepura, Nugegoda, Sri Lanka and the experiment was conducted in conformity with guidelines of the declaration of Helsinki[12].

\section{Results}

\subsection{Characteristics of Subjects}

Distributions of risk factors of the study population are given in Table 1 and 2. Among the risk factors assessed, the family history of premature heart disease showed a statistically significant association with CAD when compared to controls [OR $5.15(95 \%$ CI $2.0-12.8), \mathrm{p}=0.001]$. However, our study population did not show a significant association between family history of diabetes and dietary habits with CAD (Table 1). Results indicated that smokers had 2.22 fold significant risk, the alcoholics had 3.23 fold significant risks and interestingly, those who were classified under both alcoholics and smokers had $5.76(\mathrm{p}=0.02)$ fold risk for CAD than non-alcoholics and nonsmokers (Table 2).

Table 1. Demographic features and distribution of CAD risk factors in patients and controls.

\begin{tabular}{|c|c|c|c|c|c|c|c|}
\hline \multicolumn{2}{|l|}{ Variable } & \multicolumn{2}{|c|}{$\begin{array}{l}\text { Patients } \\
\mathrm{n} \%\end{array}$} & \multicolumn{2}{|c|}{$\begin{array}{l}\text { Controls } \\
n \%\end{array}$} & \multirow{3}{*}{$\begin{array}{l}\text { Odds } \\
\text { ratio } \\
(\mathbf{9 5 \%} \text { CI) }\end{array}$} & \multirow{3}{*}{$\begin{array}{l}\mathbf{p} \\
\text { value }\end{array}$} \\
\hline \multirow{2}{*}{ Gender } & Female & 58 & 77 & 58 & 77 & & \\
\hline & Male & 17 & 23 & 17 & 23 & & \\
\hline \multirow{4}{*}{$\begin{array}{l}\text { Family } \\
\text { history of } \\
\text { heart disease } \\
\text { Family } \\
\text { history of } \\
\text { diabetes } \\
\text { mellitus }\end{array}$} & Yes & 26 & 34.7 & 7 & 9.3 & 5.15 & \\
\hline & No & 49 & 65.3 & 68 & 90.7 & $(2.0-12.8)$ & $0.001^{\prime \prime}$ \\
\hline & Yes & 22 & 29.3 & 16 & 21.3 & 1.53 & \\
\hline & No & 53 & 70.7 & 59 & 78.7 & $(0.72-3.21)$ & 0.262 \\
\hline \multirow[b]{2}{*}{ Veganism } & Yes & 7 & 9.3 & 6 & 8.0 & 0.845 & \\
\hline & No & 68 & 90.7 & 69 & 92.0 & $\begin{array}{l}(0.27- \\
2.64)\end{array}$ & 0.772 \\
\hline
\end{tabular}

"Pearson chi square test comparing cases and controls. ${ }^{*}$ Significant at $\mathrm{p} \leq$ 0.05 .

Table 2. CAD risk estimation for smoking and alcoholism habits of male subjects in study population.

\begin{tabular}{|c|c|c|c|c|c|c|c|}
\hline & \multicolumn{2}{|c|}{$\begin{array}{l}\text { Male } \\
\text { Patients } \\
(n=58)\end{array}$} & \multicolumn{2}{|c|}{$\begin{array}{l}\text { Male } \\
\text { Controls } \\
(\mathrm{n}=\mathbf{5 8})\end{array}$} & \multirow[t]{2}{*}{$\begin{array}{l}\text { Odds } \\
\text { ratio }\end{array}$} & \multirow[t]{2}{*}{$95 \%$ CI } & \multirow[t]{2}{*}{$\begin{array}{l}p \\
\text { value }\end{array}$} \\
\hline & $\mathbf{N}$ & $\%$ & $n$ & $\%$ & & & \\
\hline $\begin{array}{l}\text { Smoking } \\
\text { Smokers }\end{array}$ & 40 & 69.0 & 29 & 50.0 & 2.22 & $1.04-4.74$ & $0.039^{*}$ \\
\hline Non-smokers & 18 & 31.0 & 29 & 50.0 & & & \\
\hline $\begin{array}{l}\text { Alcoholism } \\
\text { Alcoholics }\end{array}$ & 47 & 81.0 & 33 & 56.8 & 3.23 & $1.40-7.47$ & $0.006^{*}$ \\
\hline $\begin{array}{l}\text { Non-alcoholics } \\
\text { Behaviors }\end{array}$ & 11 & 19.0 & 25 & 43.2 & & & \\
\hline $\begin{array}{l}\text { Smoking and } \\
\text { alcoholism }\end{array}$ & 34 & 58.6 & 24 & 41.4 & 5.76 & $1.86-17.20$ & $0.002^{*}$ \\
\hline Smoking only & 6 & 10.3 & 5 & 8.7 & 4.80 & $1.03-22.37$ & $0.046^{*}$ \\
\hline $\begin{array}{l}\text { Alcoholism } \\
\text { only }\end{array}$ & 13 & 22.4 & 9 & 15.5 & 5.70 & $1.57-21.14$ & $0.008^{*}$ \\
\hline Not using any & 5 & 8.7 & 20 & 34.4 & & & \\
\hline
\end{tabular}

Smokers represent both current smokers and ex-smokers who cessation smoking more than 12 months. Alcoholics represent both regular alcoholism and former alcoholism who quite consumption of alcohol more than 12 months. ${ }^{*}$ Significant at $\mathrm{p} \leq 0.005$.

\subsection{Assessment of the Severity of Coronary Artery Disease Based on Scoring System}

Severity of coronary artery disease (CAD) assessed by the vessels score, stenosis score, and extent score by perusing the coronary angiograms of participants in the study showed a mean vessel score of 1.67 out of a maximum of 3 (95\% CI 
1.46-1.87), which indicates that the majority of the participants of this study had involvement of at least two out of the three main coronary vessels.

The mean stenosis score of the patients was 8.09 out of a theoretical maximum of $32(95 \%$ CI 6.87 - 9.30), which indicates that more than one third of the collective vessel diameter of the coronary artery segments studied was having significant stenosis. The extent score of the study population was 50.96 out of a theoretical maximum of $100(95 \% \mathrm{CI}$ 45.82 - 56.11) according to the method described by Sullivan et al 1990 [11]. Thus, the overall results of this study indicate that the severity and extent of coronary artery involvement, hence the severity of CAD in this study population is high (Table 3).

Table 3. Severity of CAD as assessed by the three scoring systems in the study population.

\begin{tabular}{|c|c|c|c|c|}
\hline & \multirow[b]{2}{*}{$\operatorname{Mean}( \pm$ SD $)$} & \multicolumn{2}{|l|}{ Range } & \multirow{2}{*}{$\begin{array}{l}95 \% \\
\text { Confidence } \\
\text { Interval }\end{array}$} \\
\hline & & Minimum & Maximum & \\
\hline Vessel score & $1.67( \pm 0.78)$ & 1 & 3 & $1.46-1.87$ \\
\hline Stenosis score & $8.09( \pm 4.76)$ & 3 & 24 & $6.87-9.30$ \\
\hline Extent score & $50.96( \pm 18.56)$ & 20 & 95 & $45.82-56.11$ \\
\hline
\end{tabular}

When the vessel score was considered $43 \%$ of patients had single vessel disease, $32 \%$ had double vessel disease and $25 \%$ had triple vessel disease. Stenosis score and extent score were classified as mild, moderate and severe diseases using scoring ranges. When the stenosis score was considered, $52 \%$ of patients had mild disease (1-7), 27\% had moderate disease $(8-15)$ and $21 \%$ severe disease $(16 \leq)$. Furthermore when the extent score was considered $15 \%$ of patients had mild disease $(\geq 25 \%), 38 \%$ had moderate disease $(26-49 \%)$ and $47 \%$ had severe disease $(\leq 50 \%)$. The angiographic characteristics of the study population have been described in detail elsewhere (13).

Table 4. Biochemical parameters between patients and controls.

\begin{tabular}{l|llll}
\hline Parameter & Mean \pm SD & Minimum & Maximum & p Value \\
\hline $\begin{array}{l}\text { Total GPX }(\mathrm{U} / \mathrm{g} \\
\text { hemoglobin) }\end{array}$ & & & & \\
Patients & $33.25 \pm 10.05$ & 13.23 & 54.31 & 0.001 \\
Controls & $43.46 \pm 5.99$ & 25.06 & 57.49 & \\
PON-1 $(\mu \mathrm{g} / \mathrm{ml})$ & & & & \\
Patients & $46.2 \pm 23.3$ & 7.9 & 114.0 & 0.001 \\
Controls & $111.7 \pm 64.0$ & 14.9 & 395.0 & \\
\hline
\end{tabular}

Independent sample $\mathrm{t}$ test comparing patients and controls. Significant at $\mathrm{p} \leq 0.05$.

\subsection{Comparison of PON-1 and GPx Activity in Patients and Control Subjects}

Independent $t$ test analysis revealed that the serum PON-1 and erythrocyte GPx activity of patients with CAD were significantly $(\mathrm{P} \leq 0.05)$ low compared to that of control subjects (Table 4). However, the serum PON-1 activity in patients with single, double and triple vessel disease in the three angiographic score groups (vessel, stenosis and extent score) were not significantly different. Interestingly, the erythrocyte GPx activity in patients with triple vessel disease was significantly low compared to single and double vessel disease indicating that erythrocyte GPx activity is a more sensitive marker of CAD compared to PON-1 (Table 5).

Table 5. Differences of PON-1, GPXactivity in subgroups of three scoring systems of severity of CAD.

\begin{tabular}{|c|c|c|c|c|}
\hline Vessel score groups & SVD & DVD & TVD & $\begin{array}{l}p \\
\text { value }\end{array}$ \\
\hline Paraoxonase-1 $(\mu \mathrm{g} / \mathrm{ml})$ & $41.0 \pm 18.2$ & $49.5 \pm 21.7$ & $51.5 \pm 35.2$ & 0.314 \\
\hline $\begin{array}{l}\text { Total GPX (U/g } \\
\text { hemoglobin) }\end{array}$ & $41.1 \pm 7.9$ & $27.9 \pm 4.2$ & $22.9 \pm 5.2$ & $0.001^{*}$ \\
\hline Stenosis score groups & (1-7) Mild & $\begin{array}{l}(8-15) \\
\text { Moderate }\end{array}$ & $\begin{array}{l}(16 \leq) \\
\text { Severe }\end{array}$ & $\begin{array}{l}\mathrm{p} \\
\text { value }\end{array}$ \\
\hline Paraoxonase $1(\mu \mathrm{g} / \mathrm{ml})$ & $43.7 \pm 19.2$ & $55.2 \pm 29.6$ & $37.8 \pm 27.3$ & 0.103 \\
\hline $\begin{array}{l}\text { Total GPX (U/g } \\
\text { hemoglobin) }\end{array}$ & $37.3 \pm 9.1$ & $28.9 \pm 8.9$ & $22.9 \pm 4.5$ & $0.001^{*}$ \\
\hline Extent score groups & $(\leq 25 \%)$ Mild & $\begin{array}{l}(26-49 \%) \\
\text { Moderate }\end{array}$ & $\begin{array}{l}(50 \% \leq) \\
\text { Severe }\end{array}$ & $\begin{array}{l}\mathrm{p} \\
\text { value }\end{array}$ \\
\hline Paraoxonase $1(\mu \mathrm{g} / \mathrm{ml})$ & $41.9 \pm 21.9$ & $43.7 \pm 17.2$ & $48.5 \pm 28.1$ & 0.657 \\
\hline $\begin{array}{l}\text { Total GPX (U/g } \\
\text { hemoglobin) }\end{array}$ & $43.6 \pm 5.8$ & $35.8 \pm 10.0$ & $30.1 \pm 9.1$ & $0.003^{*}$ \\
\hline
\end{tabular}

SVD-Single Vessel Disease, DVD-Double Vessel Disease, TVD-Triple Vessel Disease.

One way Analysis of variance (ANOVA) test performed to patients group. Significant at $\mathrm{p} \leq 0.05$ level.

\subsection{Interpretation of ROC Curves with Respect to PON-1 and GPx Activity Based on Vessel, Stenosis and Extent Score}

Multivariate logistic regression analysis was performed to eliminate influences of confounding factors for CAD. Results showed a significantly high risk of CAD associated with positive family history of CAD (odds ratio 7.81, $\mathrm{p}=0.001$ ). According to ROC analysis, subjects with cut-off value for PON-1 less than $38 \mu \mathrm{g} / \mathrm{ml}$ or GPX value less than $84.5 \mathrm{U} / \mathrm{g}$ $\mathrm{Hb}$ and positive family history of $\mathrm{CAD}$ are at high risk of development of CAD (Table 6).

Table 6. Associations of risk models for coronary artery disease in study population by multivariate logistic regression analysis.

\begin{tabular}{llll}
\hline Variable & $\begin{array}{l}\text { Odds } \\
\text { ratio }\end{array}$ & $\begin{array}{l}\text { 95\% confidence } \\
\text { interval }\end{array}$ & $\begin{array}{l}\text { p } \\
\text { value }\end{array}$ \\
\hline Age $($ Years) & 0.97 & $0.92-1.02$ & 0.269 \\
Gender & 0.81 & $0.25-2.66$ & 0.736 \\
Body mass index $\left(\mathrm{kg} / \mathrm{m}^{2}\right)^{\#}$ & 1.00 & $0.90-1.10$ & 0.998 \\
Positive Family history of CAD & 7.81 & $2.22-27.43$ & $0.001^{*}$ \\
Paraoxonase $1<38.0 \mu \mathrm{g} / \mathrm{ml}$ & 10.0 & $8.95-80.34$ & $0.050^{*}$ \\
Total GPX $<84.5 \mathrm{U} / \mathrm{g} \mathrm{Hb}$ & 16.18 & $4.56-57.36$ & $0.001^{*}$ \\
\hline
\end{tabular}

\#Consider as continuous variable *significant at $\mathrm{p} \leq 0.05$.

ROC analysis showed that the AUCs for PON-1 in predicting vessel, stenosis and extent scores was significantly low compared to that of GPX. However, the GPx showed significantly high AUC with cut-off value of $84.5 \mathrm{U} / \mathrm{gHb}$ keeping acceptable sensitivity (94\%), specificity (81\%) with positive predictive values $(84 \%)$ and negative predictive values $(93 \%)$ with a NLR of $0.06(\mathrm{AUC}=0.947)$ compared to PON-1 (AUC $=0.435$ ), suggesting that GPX was a good predictor for assessing the severity of $\mathrm{CAD}$ using vessel 
score compared to PON-1 (Table 7).

When considering the stenosis score however, GPX also showed a high sensitivity and negative predictive value (Sen $87 \%$, NP 90.3\%, NLR=0.23, AUC $=0.804$ ) which further confirms that GPx could be used in ruling out major vessel disease compared to PON-1 (Table 7).
However, GPx has shown a moderate but significantly high sensitivity and negative predictive value (Sen $71 \%$, NP $61.2 \%, \mathrm{NLR}=0.5 ; \mathrm{AUC}=0.681)$ for extent score compared to vessel score (Table 7). Thus when considering the three angiographic scores, GPx has the accuracy to rule out major coronary vessel disease.

Table 7. Receiver operating characteristic curves generated optimum cut-off values for coronary artery disease risk markers with severity of CAD scoring systems.

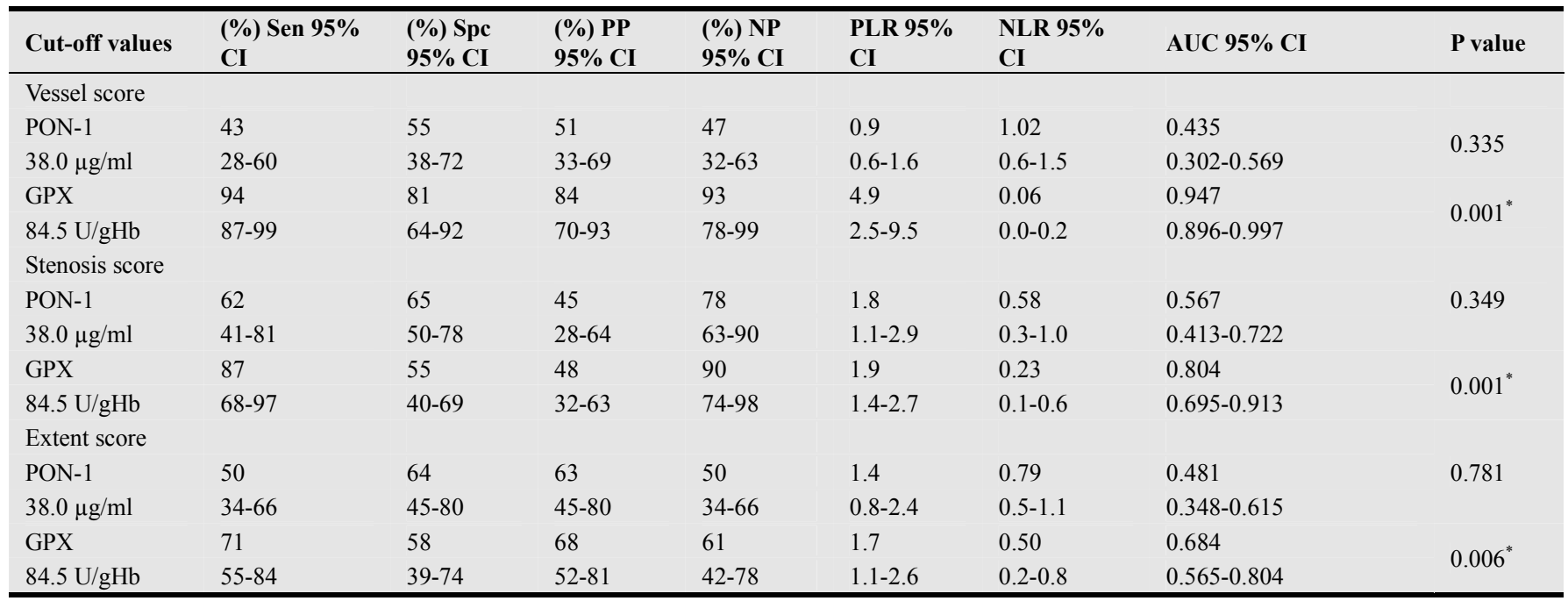

Sen- Sensitivity; Spc - Specificity; PPV - Positive predictive value; NPV - Negative predictive value; PLR- Positive likelihood ratio; NLR- Negative likelihood ratio; AUC - Area under curve; 95\% CI - 95\% confidence interval; ${ }^{*}$ Significant at $\mathrm{p}<0.05$.

\section{Discussion}

The coronary artery risk factors are not independent of one another and have direct or indirect relationships. Therefore, determining the influence of each risk factor is very important to prevent the incidence of cardiovascular diseases [14]. Among them oxidative stress has been implicated as one of the potential mechanisms of atherosclerosis. Evidence of our previous studies revealed that both GPx and PON-1 were considered to be the most sensitive markers of oxidative stress associated with vascular endothelial injury $[15,16]$. Thus, it has been hypothesized that assessment of GPx and PON-1 may be of clinical importance in predicting the accuracy of CAD. Although several studies have proved that a positive relationship exists between PON-1, GPx and CAD, these studies had limitations of assessing the extent of coronary atherosclerosis [17, 18], thus, raising doubts about the methodological process of CAD risk assessment in patients.

Although previous studies have proved that a positive relationship exist between the number of cardiovascular risk markers including PON-1 and GPx and severity of CAD, most of these studies have only concentrated on the traditional vessel score rather than the severity and extent of atheroma [17, 18]. The vessel score as described before in other studies is a traditional method of assessing the severity of ischemia compared to stenosis and extent scores. To the best of our knowledge, this is the first attempt to assess the relationship between PON-1 and GPx with extent of severity of $\mathrm{CAD}$ using three angiographic scores namely; vessel, stenosis and extent score [9-11].

The main finding of our study was that patients with triple vessel disease had significantly low GPx activity compared to double and single vessel disease indicating that low GPx activity could be one factor which influences the severity of CAD. Thus, the low erythrocyte GPx activity reported in our patients could be a consequence of increased oxidative stress induced by coronary events exacerbates the onset and severity of CAD [19]. Although our study showed low PON1 activity in patients with CAD compared to control subjects, we did not find significant differences between vessel, stenosis and extent score groups (Table 6) indicating that PON-1 has low value in predicting the accuracy of CAD compared to GPx.

Recent studies reported a strong and significant inverse association between PON-1 and CAD in Chinese population [17]. However, these studies have shown substantial study heterogeneity due to methodological limitations [18]. As our study has taken more precautions by assessing the severity of CAD using vessel, stenosis and extent scores, we believe that the cut-off value of $38.0 \mu \mathrm{g} / \mathrm{mL}$ for PON-1 and $84.5 \mathrm{U} / \mathrm{gHb}$ for GPx would be more acceptable for our South Asian study population in assessing the severity of CAD.

However, in coronary vessel based evaluation, the extent score which quantifies the proportion length of coronary endothelial surface area occluded by atherosclerosis, can be used as a strong and independent predictor for CAD. Thus, the cut-off value of $84.5 \mathrm{U} / \mathrm{gHb}$ reported for GPx with high sensitivity, specificity and negative predictive value in our 
study could be used as a potential predictor for ruling out severe CAD. Our findings further proves that erythrocyte GPx activity is a more sensitive marker for assessing the severity of CAD compared to serum PON-1 activity. Although previous studies have found that PON-1 is a potential biomarker for the severity of CAD compared to our study, such a difference could have been attributed to methodological limitations in assessing the severity of CAD $[20,21]$.

\section{Conclusions}

Present study data adds evidence to the limited data pool on contributory factors for assessment of severity of CAD using PON-1 and erythrocyte GPx activity among s South Asian population based on vessel, stenosis and extent scores which place emphasis on the number extent to which the main coronary vessels involved in luminal narrowing by atheroma. Although both PON-1 and GPx activity showed a significantly inverse relationship with the severity of CAD, angiographic scores indicated that erythrocyte GPx activity had significantly higher accuracy of ruling out major vessel disease compared to PON-1. Thus, individuals having GPx concentration of $\leq 84.5 \mathrm{U} / \mathrm{gHb}$ with positive family history of premature heart diseases are more susceptible for CAD. As GPX polymorphism may influence the response of GPX activity, more patient numbers representing different ethnic groups are needed as far as the cutoff values are concerned in predicting the accuracy for CAD.

\section{Abbreviations}

CAD: Coronary artery disease.

GPx: Glutathione peroxidase.

PON-1: Paraoxonase-1.

\section{Authors' Contributions}

DW carried out the sample collection, laboratory assays, genetic study and participated in the statistical analysis. HP has made significant contributions to conception and design, analysis and interpretation of data, drafting the manuscript. LGC has participated in study design and coordination and helped to draft the manuscript. VS participated in the patients' selection, and carried out coronary angiography. RP participated in the concept and design of the study and participated in the statistical analysis. All authors read and approved the final manuscript.

\section{Acknowledgments}

The authors thank: (i) the staff of the Cardiology Unit, Nawaloka, Hospital, Colombo and the National Hospital Sri Lanka, Colombo for providing facilities for selection of patients; (ii) Family Health Care Centre, Dept. of Family Medicine, University of Sri Jayewardenepura, Sri Lanka for assisting selection of control subjects (iii) Dr. W. A. AWijayasiri, Head of Dept. of Community Medicine,
University of Sri Jayewardenepura, Sri Lanka for the assistance with data analysis.

\section{References}

[1] Weinbrenner T, Cladellas M, Covas MI, Fito M, Tomas M, Senti M, et al. High oxidative stress in patients with stable coronary artery disease. Atherosclerosis. 2003; 168: 99-106.

[2] Dhalla NS, Temsah RM, Netticadan T. Role of oxidative stress in cardiovascular diseases. J Hypertens. 2000; 18: 655-73.

[3] Witztum JL. The oxidative hypothesis of atherosclerosis. Lancet. 1994; 344: 793-795.

[4] Blankenberg S, Rupprecht HJ, Bicket C, Torzewski M, Hafher $\mathrm{G}$, Tieret $\mathrm{L}$, et al. Glutathione peroxidase 1 acting and cardiovascular events in patients with coronary artery disease. N Engl J Med. 2003; 349 (17): 1605-13.

[5] Aviram M, Rosenblat M. Paraoxonases 1, 2, and 3, oxidative stress, and macrophage foam cell formation during atherosclerosis development. Free Radical Biology \& Medicine. 2004; 37: 1304-16.

[6] Madamanchi NR, Vendrov A, Runge MS. Oxidative stress and vascular disease. Arterioscler Thromb Vasc Biol. 2005; 25 (1): 29-38.

[7] Zachara BA, Ukleja-adamowicz M, Nartowicz E, Lecka J. Increased plasma glutathione peroxidase activity in patients with acute myocardial infarction. Med Sci Monit. 2001; 7 (3): 415-20.

[8] Gensini GG. A more meaningful scoring system for determining the severity of coronary heart disease. Am J Cardiol. 1983; 51 (3): 606.

[9] Reardon MF, Nestel PJ, Craig IH, Harper RW. Lipoprotein predictors of the severity of coronary artery disease in men and women. Circulation. 1985; 71 (5): 881-8.

[10] Hamsten A, Walldius G, Szamosi A, Dahlen G, de Faire U. Relationship of angiographically defined coronary artery disease to serum lipoproteins and apolipoproteins in young survivors of myocardial infarction. Circulation.1986; 73 (6): 1097-110.

[11] Sullivan DR, Thomas MB, Marwicks MB, Freedman B. A new method of scoring coronary angiograms to reflect extent of coronary atherosclerosis and improves correlation with major risk factors. Am Heart J. 1990; 119: 1262-6.

[12] World Medical Association. World Medical Association Declaration of Helsinki Ethical Principles for Medical Research Involving Human Subjects. JAMA. 2013; 310 (20): 2191-4.

[13] Perera PR, Wickramasinghe D, Peiris H., Chandrasena LG. and Senaratne V. Association between LDL, Apolipoprotein-B Apolipoprotein A-I and Lipoprotein(a) and Severity of Coronary Artery Disease Based on Coronary Angiography. JBM. 2015; 3, 53-61.

[14] Grundy SM, Pasternak R, Greenland P, Smith S Jr, Fuster V. Assessment of cardiovascular risk by use of multiple risk factor assessment equation. A statement for healthcare professionals from the American Heart Association and the American College of cardiology. Circulation. 1999; 100: 1481-92. 
[15] Chandrasena LG, Peiris H, Waikar HD. Biochemical changes associated with reperfusion after off-pump and on-pump coronary artery bypass graft surgery. Annl Clin lab Sci. 2009; 39 (4): $372-7$.

[16] Chandrasena LG, Peiris H, Kamani J, Wanigasuriya $P$, Jayaratne SD, Wijayasiri WA, et al. Antioxidants in patients with dengue viral infection. Southeast Asian J Trop Med Public Health. 2014; 45 (5): 1015-22.

[17] Zhou C, Cao J, Shang L, Tong $\mathrm{C}, \mathrm{Hu} \mathrm{H}$, Wang $\mathrm{H}$, et al. Reduced Paraoxonase 1 activity as a marker for severity of coronary artery disease. Disease Markers. 2013; 35: 97-103.

[18] Mateo GF, Santisteve PC, elosua R, Guallar E, Marrugat J, Bleys J, et al. Antioxidant enzyme activity and coronary heart disease: Meta-analyses of observational studies. Am J of Epidemiol. 2009; 170: 135-47.

[19] Shaikh AK, Suryakar AN. Oxidative stress and antioxidant status before and after supplementation of A-Z anti-oxidant tablets in coronary artery disease. Biomedical Research 2009; 20 (2): 136-40.

[20] Reddy ST, Devarajan A, Bourquard N, Shih D, Fogelman AM. Is it just Paraoxonase-1 or are other members of the paraoxonase gene family implicated in atherosclerosis? Curr Opin Lipidol. 2008; 19: 4005-8.

[21] Jayakumari N, Thejaseebai G. High prevalence of low serum Paraoxonase-1 in subjects with coronary Artery disease. J Clin Biochem Nutr. 2009; 45 (3): 278-84. 